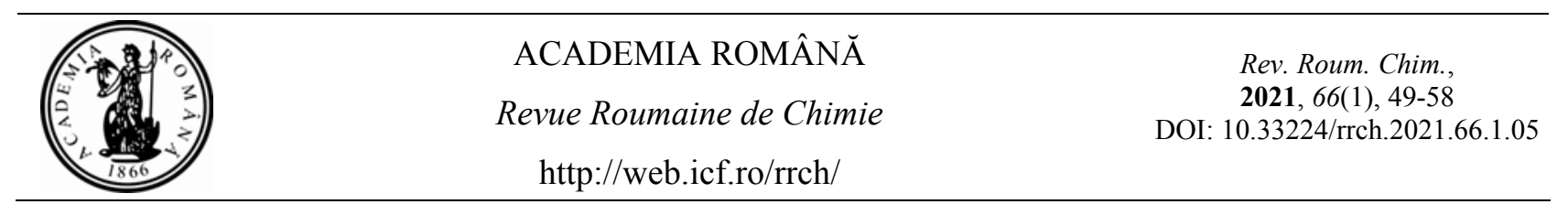

Dedicated to Professor Mihaela Hillebrand, on the occasion of her $80^{\text {th }}$ anniversary

\title{
EXPLORING THE INTERACTION \\ OF 5,6-BENZOCOUMARIN-3-CARBOXYLIC ACID \\ WITH BOVINE SERUM ALBUMIN AT THE MOLECULAR LEVEL: A BIOPHYSICAL INVESTIGATION USING MOLECULAR DYNAMICS
}

\author{
Aurica PRECUPAS ${ }^{\mathrm{a}}$ and Sorana IONESCU ${ }^{\mathrm{b}, *}$ \\ a "Ilie Murgulescu" Institute of Physical Chemistry of the Roumanian Academy, \\ Splaiul Independentei 202, 060021Bucharest, Roumania \\ ${ }^{\mathrm{b}}$ Department of Physical Chemistry, University of Bucharest, Bd. Regina Elisabeta 4-12, 030018 Bucharest, Roumania
}

In this work, molecular dynamics (MD) is used to monitor the conformational space spanned by a ligand when in the binding pocket of a protein. 5,6-benzocoumarin-3carboxylic acid was chosen as it is a relatively rigid molecule, with only one rotational degree of freedom, useful in its simplicity, whereas bovine serum albumin was used as a model protein. The initial geometry of the proteinligand complex was obtained by molecular docking. The MD simulation was carried out for $90 \mathrm{~ns}$ and the dynamic evolution of the system was explored based on the usual parameters: root mean square deviation (RMSD), radius of gyration $(\mathrm{Rg})$ and root mean square fluctuation (RMSF). The MD trajectory was analysed and interpreted in terms of the hydrogen bonds formed with the surrounding aminoacid residues and the conformational space spanned by the ligand during the interaction process.

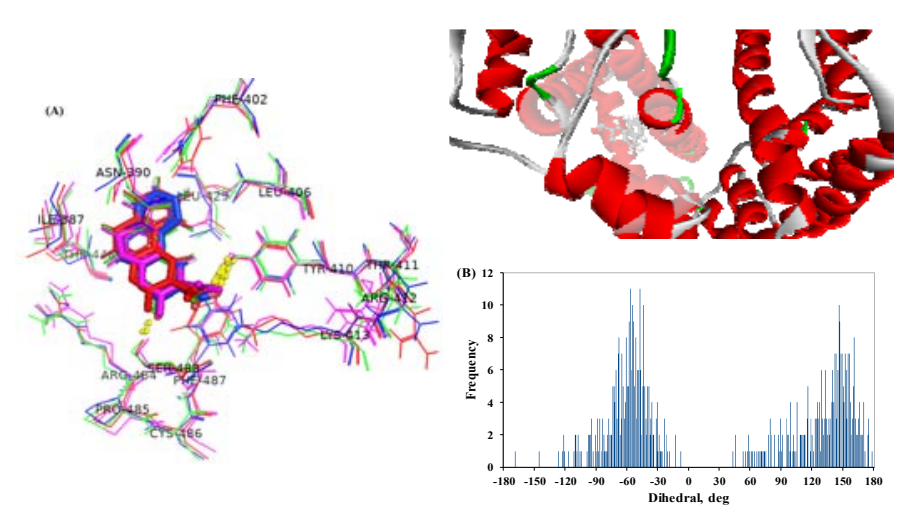

\section{INTRODUCTION}

Coumarins are heterocyclic compounds present in various plants. Coumarins and their derivatives display a vast range of biological properties, ${ }^{1}$ such as antimicrobial, ${ }^{2}$ anti-HIV ${ }^{3}$ anti-Alzheimer's disease, ${ }^{4}$ anti-cancer, ${ }^{5}$ anti-tumor, ${ }^{6,7}$ anti-oxidant, ${ }^{8,9}$ and antiinflammatory activities. ${ }^{10-12}$ Coumarin derivatives exhibit strong fluorescence in the visible region of the electromagnetic spectrum and have been studied extensively for the applications of fluorescent receptors, ${ }^{13}$ fluorescent whiteners, ${ }^{14}$ organic electroluminescent materials, ${ }^{15}$ construction of photocleavable biomaterials and bioconjugates. ${ }^{16}$

Previous studies using fluorescence spectroscopy and circular dichroism (CD) spectroscopy under physiological conditions on coumarin compounds revealed 3-carboxycoumarin interacts with human (HSA) and bovine (BSA) serum albumins, ${ }^{17}$ while the induced circular dichroism (ICD) spectra of 3-carboxycoumarin in correlation with theoretical (TDDFT) calculations have been used to obtain the

\footnotetext{
*Corresponding author: sorana@gw-chimie.math.unibuc.ro
} 
binding constants and information on the conformational changes of the ligand in the binding site of proteins. ${ }^{18}$ The photophysical properties and the behaviour in electron transfer processes of 3-carboxy-5,6-benzocoumarinic acid have been previously studied in organic solvents, in aqueous media and in the presence of aromatic amines. ${ }^{19,20}$ Theoretical and experimental study of the inclusion complexes of the 3-carboxy-5,6benzocoumarinic acid with cyclodextrins, ${ }^{21}$ or the interaction of the ligand with HSA investigated by isothermal titration calorimetry (ITC) at several temperatures $^{22}$ have been reported.

Molecular dynamics simulation is the most comprehensive computational method for predicting both fluctuations and conformational alterations of macromolecules and molecular mechanisms of interaction between proteins and ligands. Molecular dynamics simulation has been used to give insight into amyloid oligomer formation process ${ }^{23}$ and destabilization effect of small molecules on the preformed fibril and oligomer ${ }^{24-26}$ providing important role in the designing of new inhibitors. Binding of different classes of ligands to various serum albumin binding sites $\mathrm{s}^{27,28}$ has been revealed by molecular docking, while the binding forces, the protein structural stability and conformational changes upon interaction have been evaluated via molecular dynamics analysis in the simulated environment. ${ }^{29-32}$

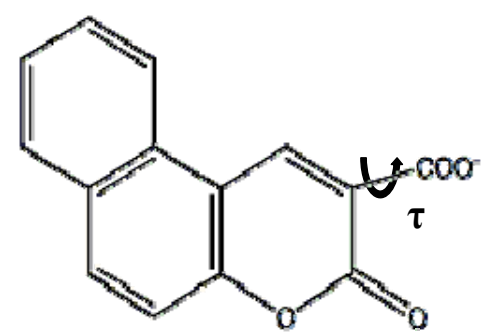

Scheme 1 - Molecular structure and rotation dihedral of 5,6-benzocoumarin-3-carboxylic acid.

5,6-benzocoumarin-3-carboxylic acid (BCCA) is a relatively rigid molecule, with only one rotational degree of freedom (see Scheme 1). As such it may be useful in understanding interactions between small molecules and the chiral medium of a protein at the molecular level and the dynamics of conformational changes induced by the binding process. This is relevant in understanding conformational dependent properties such as the induced circular dichroism (ICD) signal that arises for small molecules within the chiral medium of a protein at the molecular level. In this case, the static approach to the binding process may prove incomplete and a broader view on the conformational space of the ligand and the surrounding aminoacids during interaction becomes necessary. To this end, molecular dynamics was used to monitor the time evolution of binding and the conformational space spanned by the ligand during interaction.

\section{Molecular Dynamics procedure}

The crystal structure of BSA (PDB ID: 4F5S) ${ }^{33}$ was taken from the RCSB Protein Databank. The molecular geometry optimization of BCCA structure was obtained using density functional theory with B3LYP functional and 6-31G* basis set by Gaussian09. ${ }^{34}$ AutoDock Vina program ${ }^{35}$ was used to simulate the binding conformation of BCCA with BSA. Water molecules were removed and polar hydrogen atoms were added followed by the calculation of Gasteiger charges. The Lamarckian genetic algorithm (LGA) was applied for local optimization of molecular complexes. MGL Tools ${ }^{36}$ was used to convert ligand and protein structure to pdbqt format. The rotatable bond of the ligand structure was automatically detected and assigned. Autodock blind docking was carried out to identify the possible binding site of BCCA within the 3D geometry of BSA. In a first step, the protein structure was held rigid and the grid step was set at $1 \AA$. Docking was carried out setting the grid sizes of the three axes ( $x, y$ and z) to $40 \times 40 \times 48$ points. Ten docked conformations were generated for each docking calculation, according to their score function. Since the lowest binding energy conformer was obtained for BCCA binding at site II of BSA, in a second step, molecular docking was performed considering flexible aminoacid residues from this binding site of the protein. A search space was defined to enclose the active site of protein (site II) with the box dimension of $22 \times 22 \times 20 \AA(\mathrm{x}, \mathrm{y}$ and $\mathrm{z}$ values) and a grid point spacing of $1 \AA$. The complex with the highest score function was examined by BIOVIA Discovery studio $2019^{37}$ (http://accelrys.com/products/discovery-studio). Gromacs 2019 sofware ${ }^{38,39}$ was used to carry out MD simulations for native BSA and BSA-BCCA complex using GROMOS96 54a7 force field. ${ }^{40}$ The structure of the lowest binding energy conformer of BCCA at site II of BSA obtained from molecular docking study was used as the starting conformation for MD simulation. The topology of BCCA was obtained from the 
Automated force field Topology Builder ${ }^{41}$ (ATB, http://compbio.biosci.uq.edu.au/atb). The system was positioned in a cubic periodic box and the distance between the outer surface of the protein and the box walls was set to at least $1 \mathrm{~nm}$. The single-point charge $3(\mathrm{SPC} 3)^{42}$ model for water molecules was used and $\mathrm{Na}^{+}$counter ions were added for maintaining the overall charge neutrality of the system. The prepared system was equilibrated at $300 \mathrm{~K}$ with modified Berendsen thermostat and 1 bar with Parrinello-Rahman barostat $^{43}$ in successive steps after energy minimization. Particle Mesh Ewald (PME) ${ }^{44}$ method was used in the analysis of long-range electrostatic interactions and for non-bonded interactions, a cut-off of $1 \mathrm{~nm}$ was used. A MD simulation of $90 \mathrm{~ns}$ was run at a 2 femtosecond (fs) time step and coordinates were saved every 100 picosecond (ps) for further analysis. Molecular graphics were created using Discovery Studio and PyMol. ${ }^{45}$ The UCSF Chimera package ${ }^{46}$ from the Resource for Biocomputing, Visualization, and Informatics at the University of California, San Francisco (supported by NIH P41 RR-01081) was used to analyse the MD trajectories. The parameters such as root mean square deviation (RMSD), root mean square fluctuation (RMSF) and the radius of gyration $(\mathrm{Rg})$ were calculated in order to assess the timescale of reaching equilibrium. Then the ligand-protein interaction was monitored by determining the number of hydrogen bonds that the ligand forms with neighbouring aminoacid residues and their time evolution. Snapshots of 5 randomly selected conformations characterized by the most frequent $\mathrm{H}$ bonds were built and aligned using PyMol.

The conformational space spanned by the ligand was analysed by building histograms for each value of the dihedral that characterizes the rotation of the $\mathrm{COO}^{-}$group relative to the aromatic fragment. A free energy vs. dihedral profile corresponding to the bound ligand was obtained from these histograms, according to eq. (1).

$$
\Delta \mathrm{G}=-\mathrm{RT} \ln \mathrm{P}
$$

where $\mathrm{P}$ is the Boltzmann fraction of each conformation.

The section through the potential energy surface along the carboxyl rotation dihedral for the isolated ligand molecule was built up in Gaussian09, with the PBE0 functional and a $6-311++\mathrm{G}(2 \mathrm{~d}, 2 \mathrm{p})$ basis set. The dihedral was frozen with a step of $10 \mathrm{deg}$, while all the other coordinates were allowed to relax. Solvation was simulated in the frame of the PCM model (solvent-water).

\section{RESULTS AND DISCUSSION}

Molecular docking of BCCA with protein offers insight into the preferred binding site and a rough estimation of the binding conformation. The lowest binding energy conformer $(-8.5 \mathrm{kcal} / \mathrm{mol})$ obtained from molecular docking at site Sudlow II of protein is twisted, with a value of $-56.48 \mathrm{deg}$ for the $\mathrm{COO}^{-}$group torsion angle $(\tau)$ in respect with the planar $\pi$ system. BCCA is located in a cavity (Figure 1) surrounded by several hydrophobic aminoacids (Val432, Leu452, Leu429, Leu406, Leu386) and forms hydrogen bonds with Asn390 and Ser488. In addition, electrostatic interactions between the $\mathrm{NH}_{3}^{+}$group of LYS413 and the carboxyl in BCCA and $\pi$-cation interactions between the $\mathrm{NH}_{2}^{+}$group of Arg409 and the $\pi$ electron system of the ligand could be observed, along with van der Waals forces established with Tyr410.

The MD simulation was used to further investigate the BSA-BCCA interaction at the molecular level in solution and the possible structural changes of BSA and the ligand induced by the binding process. The time evolution of the system was monitored by plotting specific parameters along the timescale of 0 to 90 ns. Plots of RMSD for all $\mathrm{C} \alpha$ atoms in BSA and the BSABCCA complex calculated with respect to the initial structure as a frame reference are shown in Figure 2A. Equilibrium is reached after approximately $60 \mathrm{~ns}$ of simulation. The average RMSD values for native BSA and the BSA-BCCA complex at equilibrium were $0.61 \pm 0.03 \mathrm{~nm}$ and $0.58 \pm 0.04 \mathrm{~nm}$, respectively, so only a small structural change is induced in the protein subsequent to ligand binding.

$\mathrm{Rg}$ is a measure of the level of protein compactness. The calculated $\mathrm{Rg}$ values of native BSA and protein-ligand complex displayed in Figure 2B were stabilized after 70 ns. The average values of $\mathrm{Rg}$ for both systems calculated between $70 \mathrm{~ns}$ and 80 ns were approximately similar, $2.77 \pm 0.01 \mathrm{~nm}$ and $2.78 \pm 0.01 \mathrm{~nm}$, respectively. The result suggested that the compactness of protein was unchanged by binding. The time dependent change of SASA (Figure 2C) showed a similar evolution, only slower, due to binding, comparing to that for native BSA. The protein becomes more compact in time, as SASA decreases in two steps. It first decreases for $20 \mathrm{~ns}$, remains approximately constant up to $50 \mathrm{~ns}$ (SASA average value is $301.05 \pm 3.45 \mathrm{~nm}^{2}$ ), then decreases again for native BSA $\left(292.88 \pm 3.71 \mathrm{~nm}^{2}\right)$, while for the complex it is constant for another $15 \mathrm{~ns}$, 
up to approximately $65 \mathrm{~ns}\left(300.15 \pm 3.53 \mathrm{~nm}^{2}\right)$, then decreases and reaches approximately the same value $\left(293.84 \pm 3.81 \mathrm{~nm}^{2}\right)$ as for native BSA $(291.78 \pm$ $4.16 \mathrm{~nm}^{2}$ ) at equilibrium. So, the protein was gradually compacted, the process being slower in presence of the ligand.

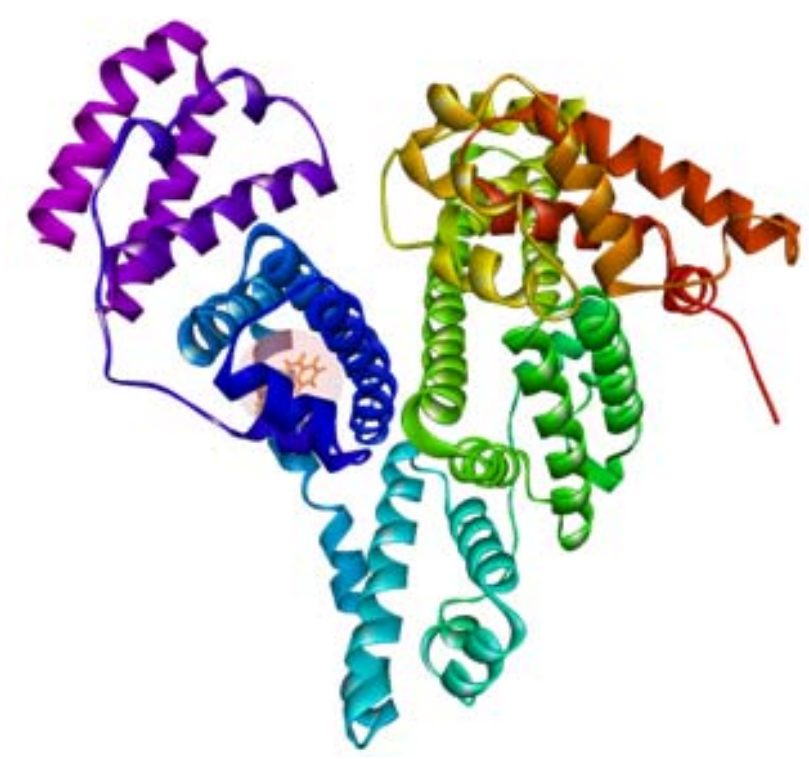

Fig. 1 - The predicted conformation of BSA-BCCA complex with the lowest docking energy. BSA was displayed in cartoon; BCCA was shown using sticks.
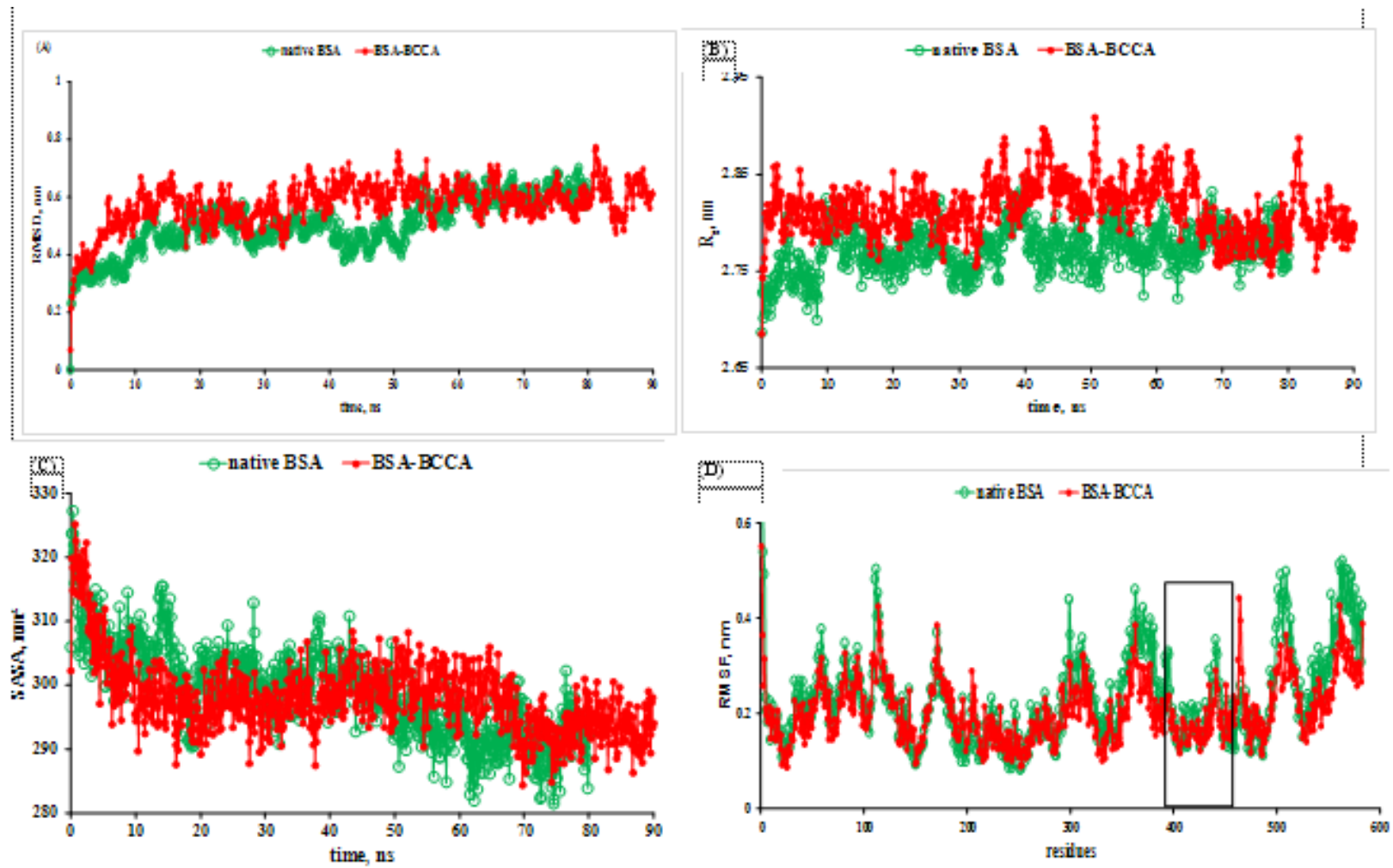

Fig. 2 - (A) Time dependence of the root mean square deviations (RMSD), (B) Time evolution of the radius of gyration (Rg) for native BSA and BSA-BCCA complex, (C) The plot of solvent accessible surface area (SASA) versus time for native BSA and BSABCCA complex, (D) the RMSF values as a function of residue numbers, the residues located in binding pocket highlighted by box. 
The RMSF values were calculated and plotted (Figure 2D) to evaluate the fluctuations of aminoacid residues. Some fluctuations were observed for both systems. The highlighted region (site Sudlow II) presents less atomic fluctuation. Thus, it indicated that the residues located in the binding site looked more rigid as a result of the binding to BCCA.

The main interest lays with the ligand and its binding dynamic features at the molecular level. The MD trajectory was analysed in terms of the $\mathrm{H}$ bonds it forms with the aminoacid residues within site Sudlow II and the conformation it adopts in the chiral relatively rigid environment of the protein. The Gromacs criteria for $\mathrm{H}$ bond formation used were: the donor-acceptor distance is not greater than $0.35 \mathrm{~nm}$ and the hydrogen - donor-acceptor angle has a maximum value of $30 \mathrm{deg}$. Data was collected every $100 \mathrm{ps}$. The time dependence of hydrogen bonds between protein and ligand is presented in Figure 3.
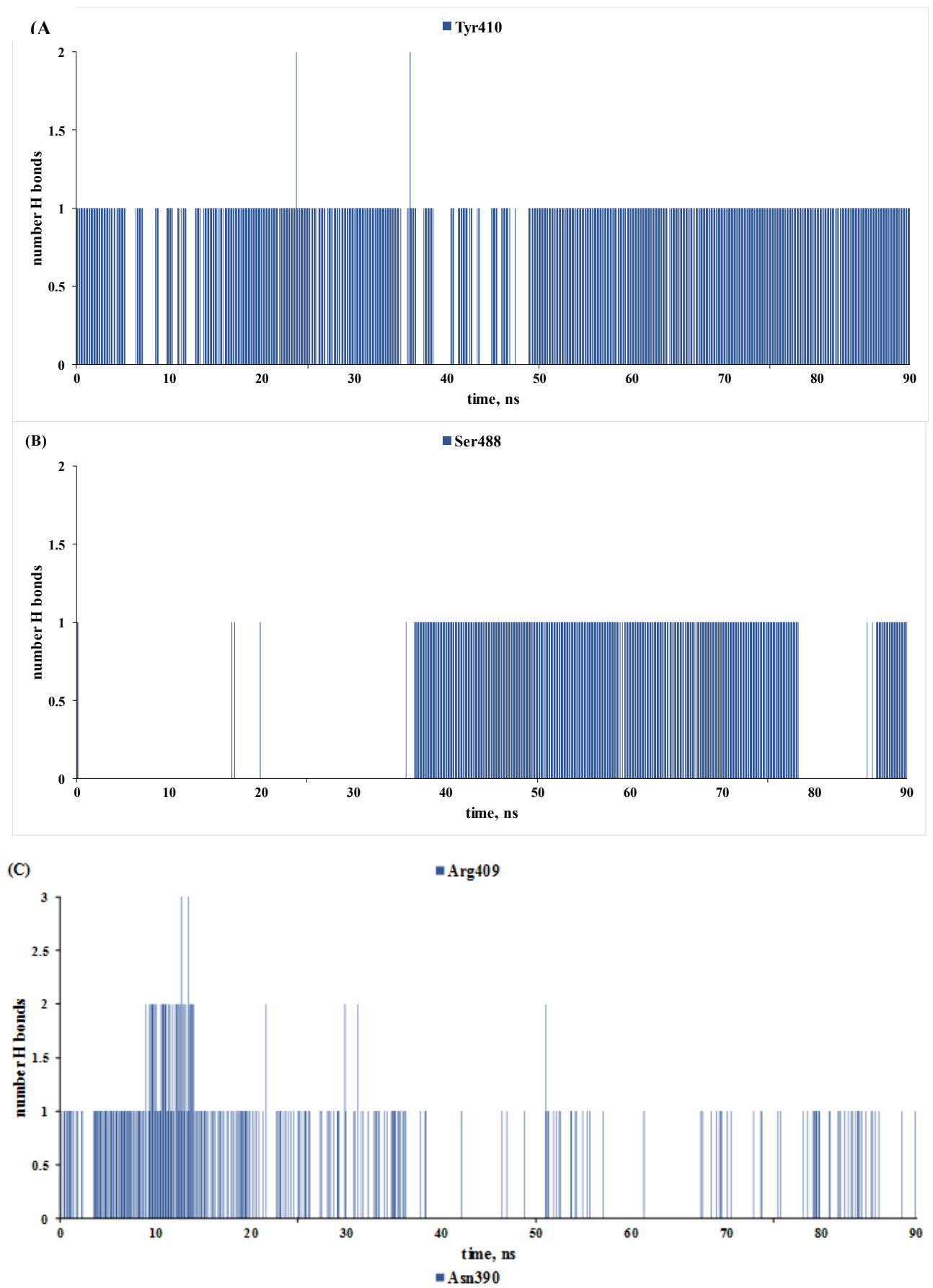

Fig. 3 - Number of hydrogen bonds between BCCA and aminoacid residues from the binding site in time A) Tyr410; B) Ser488; C) Arg409; D) Asn390; E) Lys413; F) Total. 


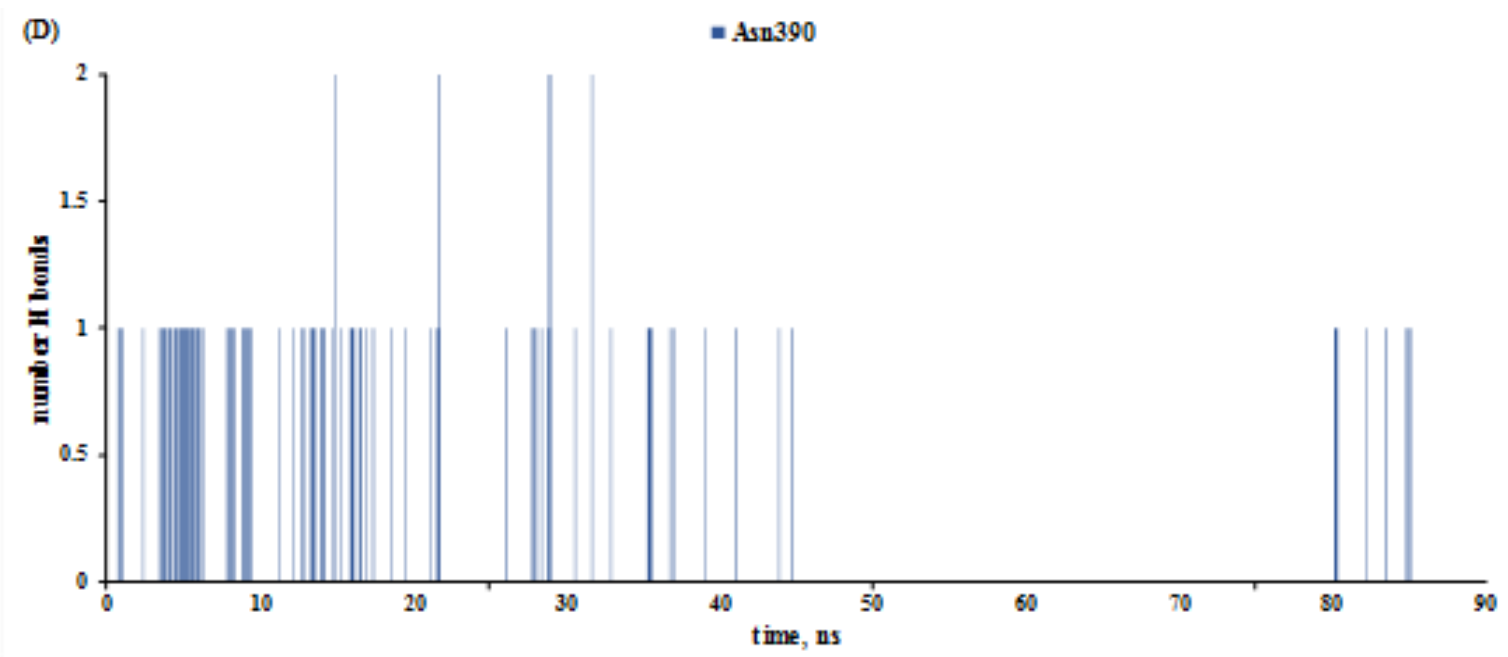

(E)

- Lys 413

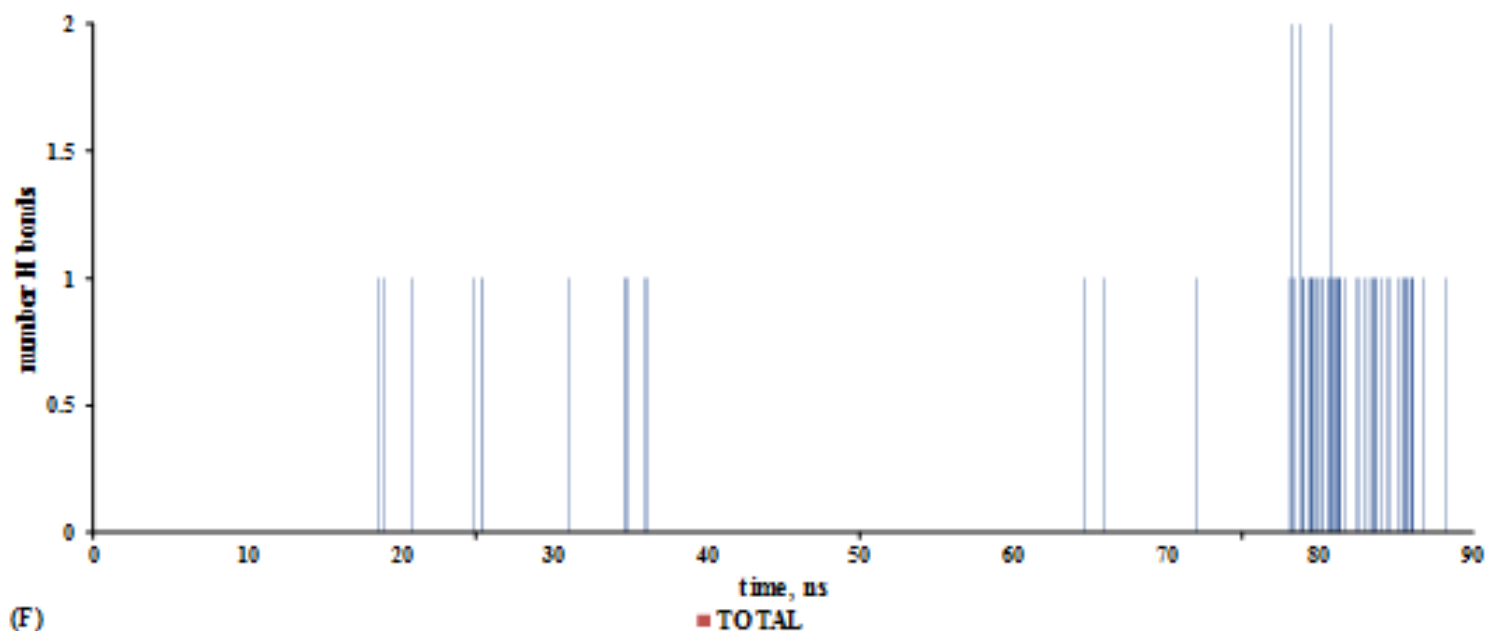

(F)

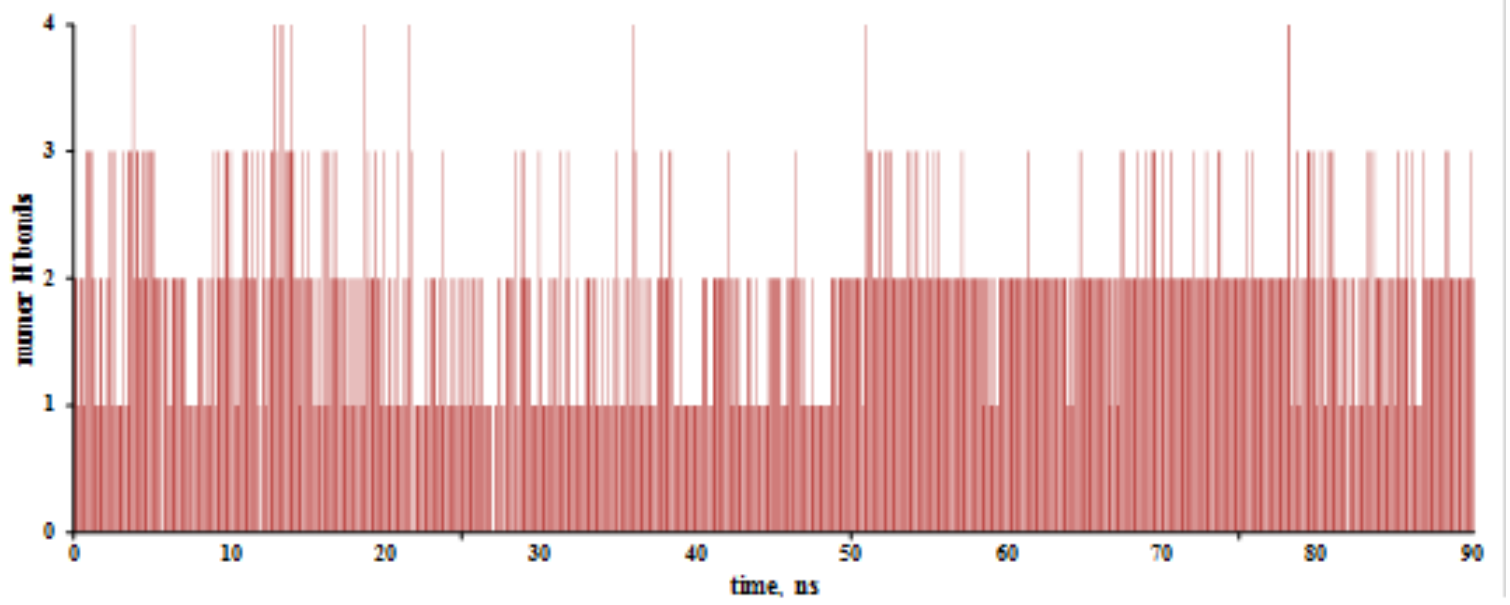

Fig. 3 (continued).

The most abundant interaction pose is the one in which $2 \mathrm{H}$ bonds are formed and its frequency rises after $50 \mathrm{~ns}$. There are only 5 residues with which BCCA forms $\mathrm{H}$ bonds, i.e. Asn390, Arg409, Tyr410, Lys413, Ser488. Among these, the most frequent ones are with Tyr410 (Figure 3A) and
Ser488 (Figure 3B), meaning that docking does not predict the exact orientation of the ligand within the binding site and the actual interactions in detail, and the MD simulation is necessary for a rigorous insight, as expected. While the $\mathrm{H}$ bond with Tyr410 is found on the whole period of the 
simulation, it is obvious that the one with Ser488 appears after about $36 \mathrm{~ns}$ since the start of the simulation and is practically absent again from 78 to 86 ns. Arg409 (Figure 3C) forms one $\mathrm{H}$ bond with the ligand as well, but especially up to $36 \mathrm{~ns}$, then drops in frequency. Asn390 (Figure 3D) forms one $\mathrm{H}$ bond more frequently, that disappears at the end of simulation time, while Lys413 (Figure 3E) is active in forming one $\mathrm{H}$ bond during the last nanoseconds of the simulation, at equilibrium. One can see that even in the equilibrium period there is a dynamics of the protein-ligand interaction at the level of the $\mathrm{H}$ bonds formed with the neighbouring aminoacids (Figure 3F).

Histograms were built up in order to monitor $\mathrm{H}$ bond number and frequency in time, overall and along the two main steps of the process, i.e. during equilibration and at equilibrium. We also monitored the aminoacids that formed $\mathrm{H}$ bonds with BCCA in Sudlow site II for both any number of $\mathrm{H}$ bonds formed and the poses in which the most frequent number of $\mathrm{H}$ bonds are formed, i.e. two $\mathrm{H}$ bonds.

Again, data in Figure 4A indicate that 2 is the most frequent number of $\mathrm{H}$ bonds formed by the ligand, as stated before. The percent of its frequency increases at equilibrium to $78 \%$. Tyr410 acts as a proton donor, the BCCA carboxylate group being the acceptor in the most frequent $\mathrm{H}$ bond formed. As it can be seen in Figures 4B and $4 \mathrm{C}$, the percent of this $\mathrm{H}$ bond during the entire period of the simulation is $84 \%$ and $92 \%$ from the poses in which $2 \mathrm{H}$ bonds are formed between BCCA and the surrounding aminoacids, while at equilibrium it rises to $98 \%$ overall and $100 \%$ from the $2 \mathrm{H}$ bonds pose.

The second most important $\mathrm{H}$ bond is the one between Ser488 and the carbonyl in BCCA. Although it is established rather late in the simulation (approximately after $36 \mathrm{~ns}$ ), it has a large frequency at equilibrium, with $72 \%$ of the overall conformations and $81 \%$ form the ones in which $2 \mathrm{H}$ bonds are formed. It is, nonetheless a more labile $\mathrm{H}$ bond, as its frequency is very low at equilibrium for about $8 \mathrm{~ns}$, from 78 to $86 \mathrm{~ns}$ since the starting time. A review of the $\mathrm{H}$ bonds formed during this period reveals that Ser488 is mainly replaced by Lys 413 as the second $\mathrm{H}$ bond donor in interaction with the carboxyl group in BCCA.

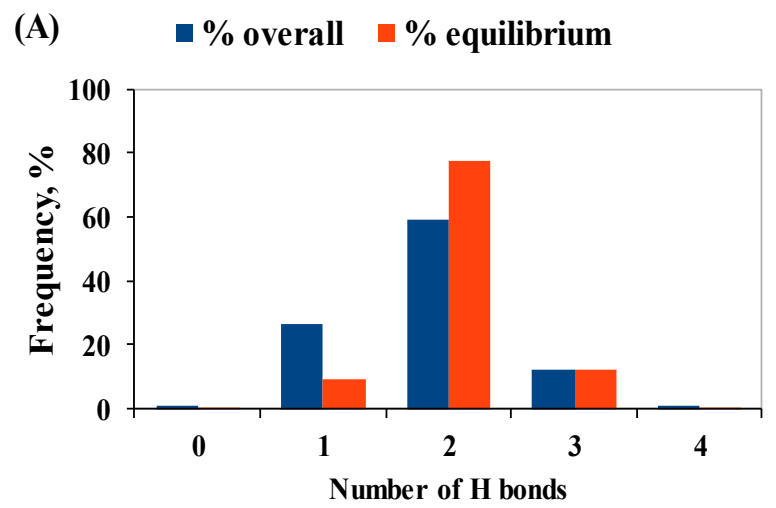

(B)
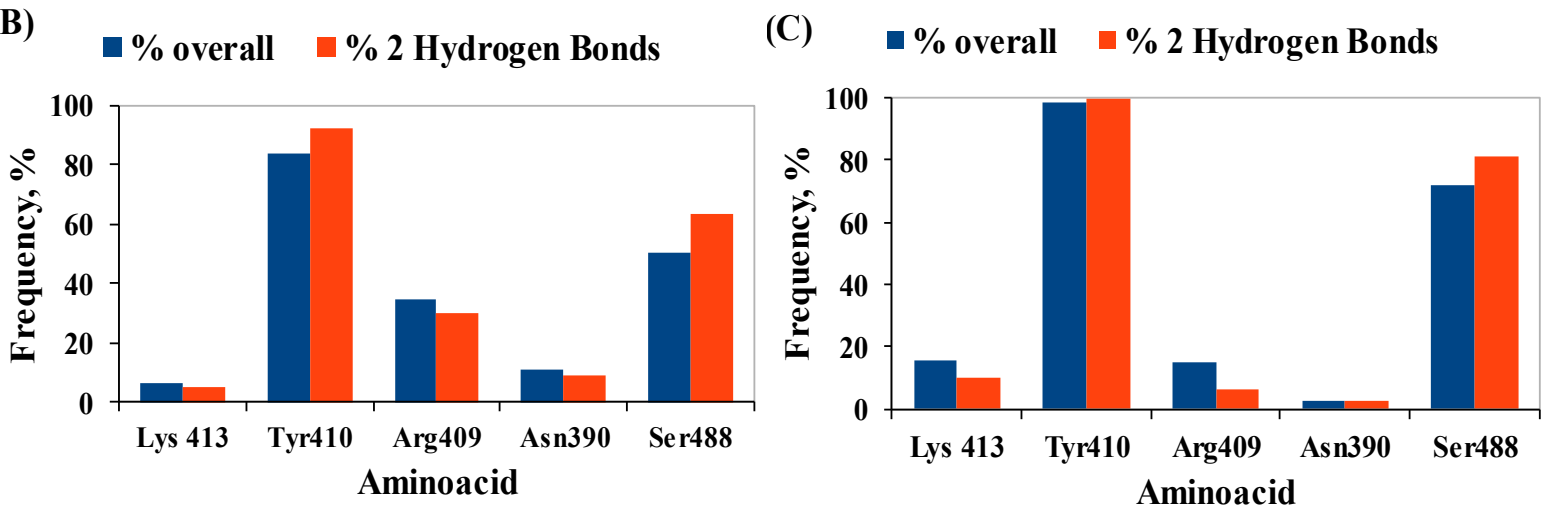

Fig. 4 - A) Histogram of the total number of BCCA-aminoacid $\mathrm{H}$ bonds along the entire timescale of the simulation (0-90 ns) and at equilibrium (60-90 ns). B and C) Histogram showing the frequency with which aminoacids form $\mathrm{H}$ bonds overall or when $2 \mathrm{H}$ bonds are formed during B) the entire period of the simulation and C) at equilibrium. 

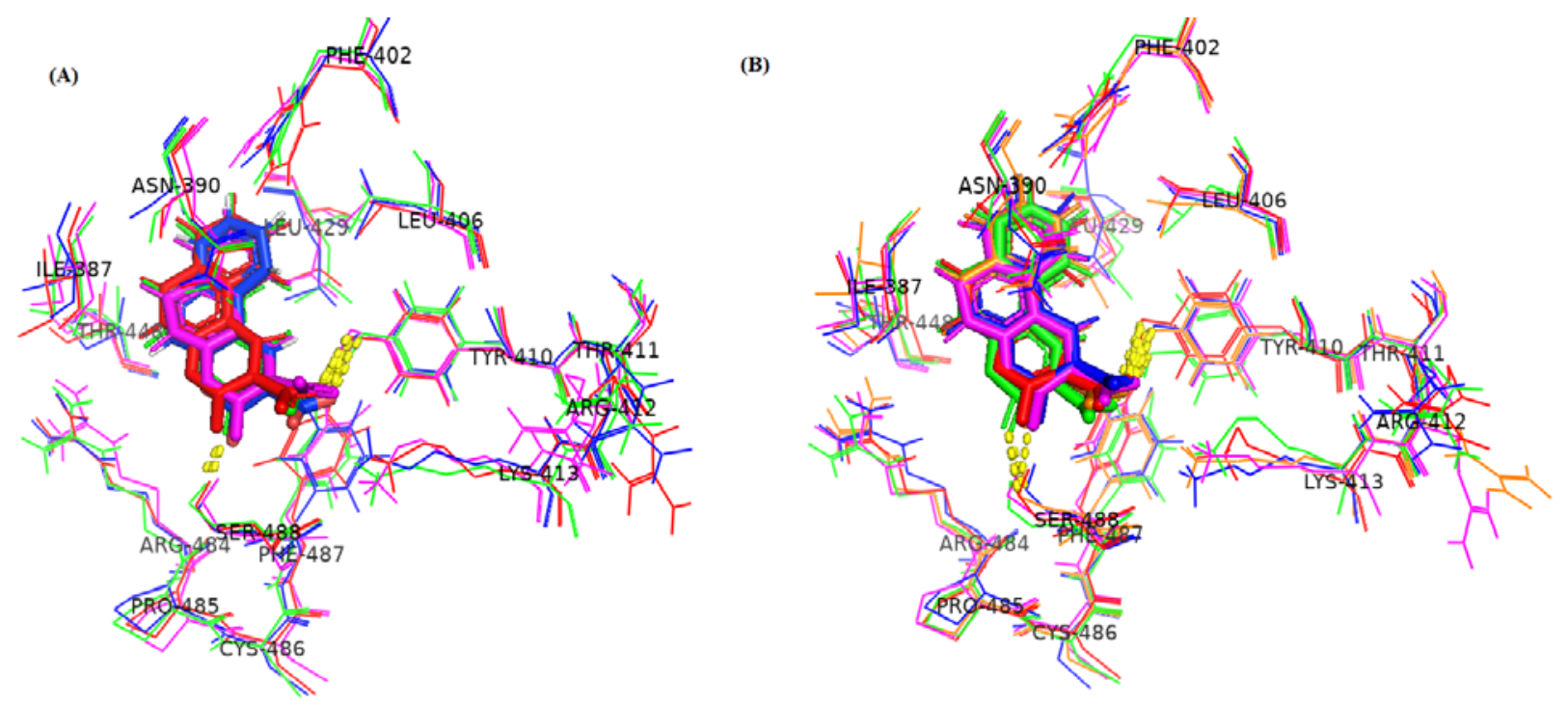

Fig. 5 - Overlap of 5 randomly chosen snapshots with the most frequent $2 \mathrm{H}$ bonds formed for A) the equilibration period and B) at equilibrium. The hydrogen bonds are displayed by yellow dotted lines, the aminoacid residues are presented by lines and the ligand is displayed by sticks.
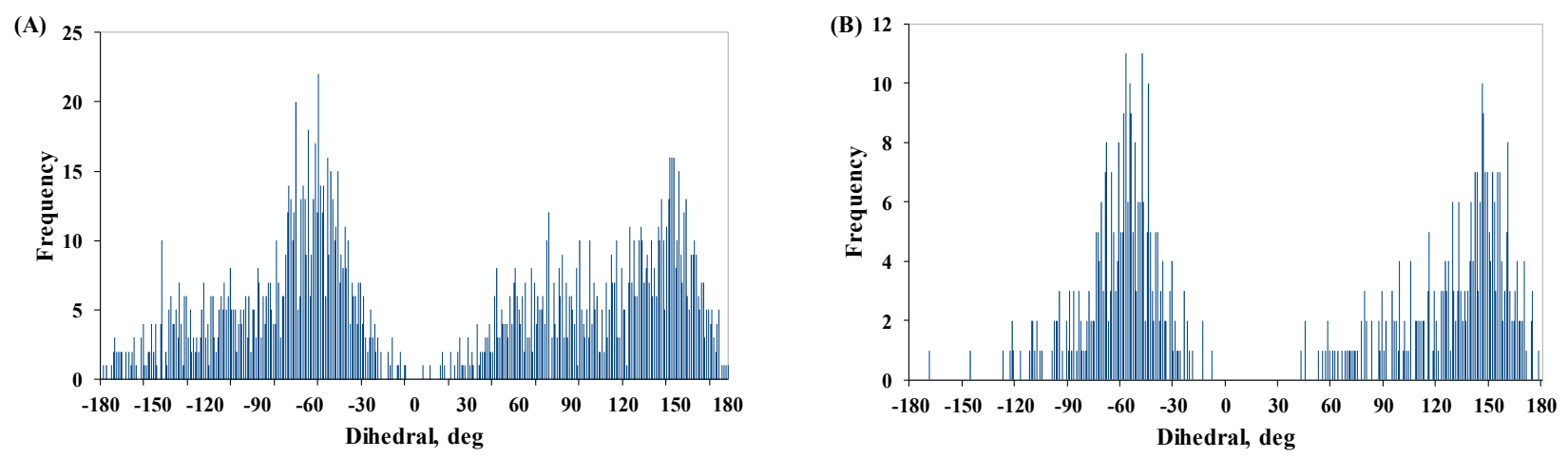

Fig. 6 - Histograms for rotation dihedral A) on the entire period of simulation, B) at equilibrium.

In order to have an insight on the conformational aspects of the protein-ligand interaction and the conformational space spanned by the ligand, 5 randomly selected snapshots from the equilibration period and at equilibrium were aligned and overlapped in Figure 5. The snapshots were selected from the most frequent pose encountered, i.e. when BCCA forms $2 \mathrm{H}$ bonds with Tyr410 and Ser488. The alignment was done relative to the $\mathrm{C} \alpha$ atom of each aminoacid residue at less than $3.5 \AA$ from the ligand.

As it can be seen from Figure 5, there are no important conformational changes of the surrounding aminoacids and the ligand, except for the carbonyl rotation dihedral, which takes the values of $-52 \pm 12 \mathrm{deg}$ or $148 \pm 11 \mathrm{deg}$. So even at equilibrium, the static picture of binding may be incomplete, especially in what concerns properties which are highly conformation dependent.
Another aspect we explored in this study is changes in the conformational space spanned by the ligand due to binding. The only rotational degree of freedom of the molecule is rotation of the carboxyl group relative to the extended aromatic system. The histograms on the entire period of the simulation and at equilibrium are presented in Figure 6.

The most frequent dihedral values are $-55 \mathrm{deg}$ and $148 \mathrm{deg}$ overall, and $-57 \mathrm{deg}$ and $146 \mathrm{deg}$ at equilibrium. The most frequent conformations do not change at equilibrium, but frequency of the quasi-planar conformations drops, so that the number of populated conformational states decreases at equilibrium. We built up the section through the potential energy surface along this dihedral for the isolated molecule of BCCA in water and it is symmetric, with minima at $90 \mathrm{deg}$ and $-90 \mathrm{deg}$ and a barrier to rotation of $1 \mathrm{kcal} / \mathrm{mol}$ (Figure 7A). 

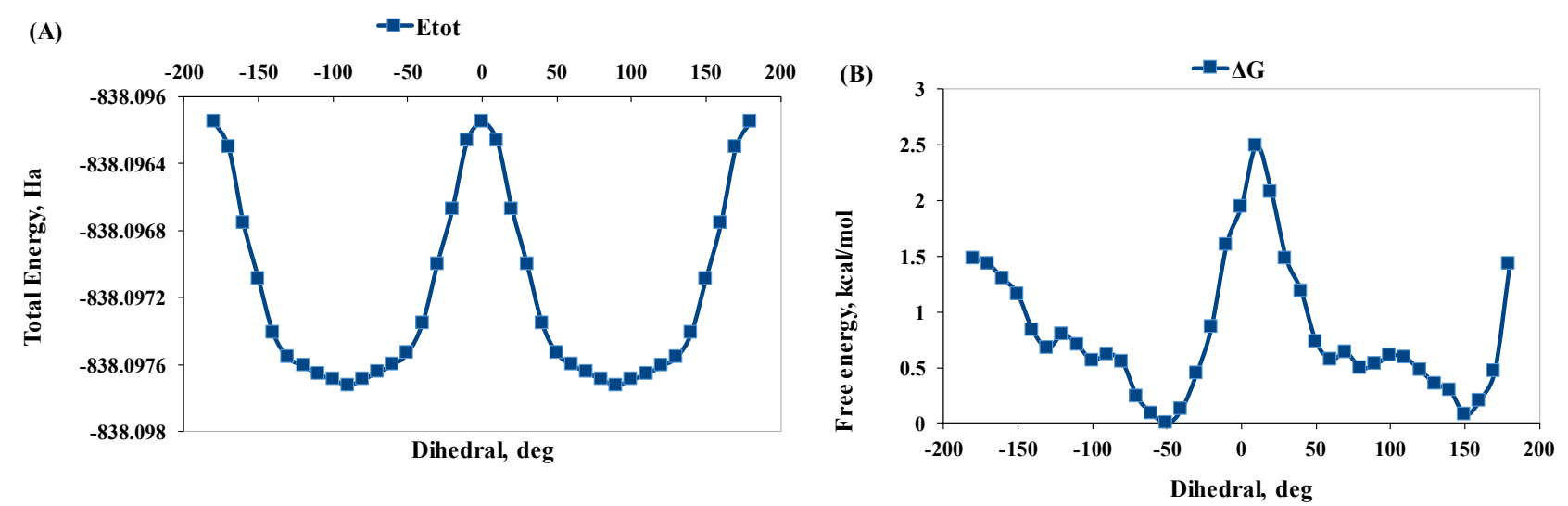

Fig. 7 - Energy profile vs. the $\mathrm{COO}^{-}$rotation dihedral. A) Total energy for the isolated BCCA molecule in water, B) frequency histogram converted to free energy profile from the MD simulation.

The free energy profile (Figure 7B) obtained from the Boltzmann populations of each dihedral value, with a step of $10 \mathrm{deg}$ is not symmetric, due to the interaction to the relatively rigid and chiral medium of the binding site. Minima are obtained for $-50 \mathrm{deg}$ and $150 \mathrm{deg}$ with shallow minima for $130 \mathrm{deg}$ and $80 \mathrm{deg}$. This comes as a completion of results from the selected snapshots above. Although maxima correspond to planar geometries, as for the isolated molecule, minima are different and so torsional states of the molecule have different populations subsequent to binding. For studying any property highly dependent on conformational changes MD simulations may be needed in order to properly explain experimental data.

\section{CONCLUSIONS}

MD simulations were successfully applied to a model protein-ligand binding system, with the aim of highlighting the dynamics of the $\mathrm{H}$ bonds the ligand forms with the surrounding aminoacids and the conformational space of the ligand. Two $\mathrm{H}$ bonds are the most frequent formed ones, especially at equilibrium, i.e. Tyr410 with the carboxylate fragment and Ser488 with the carbonyl. The population of conformational states of the ligand needs thorough investigation, as important changes were found passing from the isolated ligand molecule to the bound ligand. While the isolated BCCA has a twisted geometry with a dihedral of $\pm 90 \mathrm{deg}$, the bound ligand is restricted by the interaction with the rigid medium to dihedral values of $-57 \mathrm{deg}$ and $146 \mathrm{deg}$.

It is apparent that in the case of protein-ligand interactions dynamics at equilibrium is important and several tens of nanoseconds may be needed in order to properly average the properties of interest and correctly describe the statistics of the system.

Acknowledgements. The authors wish to thank Dr. Daniel Angelescu from the Institute of Physical Chemistry of the Romanian Academy for access to MD facilities.

\section{REFERENCES}

1. X. M. Peng, G.L. Damu and C. Zhou, Curr. Pharm. Des., 2013, 19, 3884-3930.

2. R. Kharb, M. Kaur and A.K. Sharma, Int. J. Pharm. Sci. Rev. Res., 2013, 20, 87-94.

3. I. Kostova, S. Raleva, P. Genova and R. Argirova, Bioinorg. Chem. Appl., 2006, 1-9.

4. N. Jiang, Q. Huang, J. Liu, N. Liang, Q. Li, Q. Li and S. S. Xie, Eur. J. Med. Chem., 2018, 146, 287-298.

5. J. Klenkar and M. Molnar, J. Chem. Pharm. Res., 2015, 7, 1223-1238.

6. A. Thakur, R. Singla and V. Jaitak, Eur. J. Med. Chem., 2015, 101, 476-495.

7. Y. Guo, Y. Wang, H. Li, K. Wang, Q. Wan, J. Li, Y. Zhou and Y. Chen, ACS. Med. Chem. Lett., 2018, 9, 502-506.

8. I. Najmanova, M. Dosedel, R. Hrdina, P. Anzenbacher, T. Filipsky, M. Riha and P. Mladenka, Curr. Top. Med. Chem., 2015, 15, 830-849.

9. Y. Al-Majedy, A. Al-Amiery, A. Kadhum and A. Mohamad, Sys. Rev. Pharm., 2017, 8, 24-30.

10. Y. Bansal, P. Sethi and G. Bansal, Med. Chem. Res., 2013, 22, 3049-3060.

11. M. B. Nayeli, H. R. Maribel, J. F. Enrique, B. P. Rafael, A. F. Margarita, F. M. Macrina, M. D. Ivan and G. C. Manasés, Nat. Prod. Res., 2019, 1-5.

12. B. M. Chougala, S. Samundeeswari, M. Holiyachi, N. S. Naik, L. A. Shastri, S. Dodamani, S. Jalalpure, S. R. Dixit, S. D. Joshi and V. A. Sunagar, Eur. J. Med. Chem., 2018, 143, 1744-1756.

13. Y. Shiraishi, N. Hayashi, M. Nakahata, S. Sakaic and T. Hiraia, RSC Adv., 2017, 7, 32304-32309.

14. I. H. Leaver and B. Milligan, Dyes Pigm., 1984, 5, 109-144.

15. T. Z. Yu, P. Zhang, Y. L. Zhao, H. Zhang, J. Meng, D. W. Fan, L. L. Chen and Y. Q. Qiu, Org. Electron., 2010, $11,41-49$. 
16. W. Shen, J. Zheng, Z. Zhou and D. Zhang, Acta Biomat., 2020, 115, 75-91.

17. A. Varlan and M. Hillebrand, Open Chemistry, 2011, 9, 624-634.

18. A. Varlan and M. Hillebrand, J. Mol. Struct., 2013, 1036, 341-349.

19. C. Tablet, A. Jelea and M. Hillebrand, J. Photochem. Photobiol. A: Chem., 2006, 183, 89-97.

20. C. Tablet and M. Hillebrand, J. Photochem. Photobiol. A: Chem., 2007, 189, 73-79.

21. C. Tablet and M. Hillebrand, Spectrochim. Acta A Mol. Biomol. Spectrosc., 2008, 70, 740-748.

22. R. Sandu and M. Hillebrand, Rev. Roum. Chim., 2012, 57, 421-426.

23. B. Ma and R. Nussinov, Protein Sci., 2002, 1, 23352350 .

24. W. M. Berhanu and A. E. Masunov, Biophys. Chem., 2010, 149, 12-21.

25. Q. Wang, L. Ning, Y. Niu, H. Liu and X. Yao, J. Phys. Chem. B, 2015, 119, 15-24.

26. J. A. Lemkul and D. R. Bevan, Biochemistry, 2010, 49, 3935-3946.

27. A. Precupas, R. Sandu and V. T. Popa, J. Phys. Chem. B, 2016, 120, 9362-9375.

28. A. Precupas, R. Sandu, A. R. Leonties, D.-F. Anghel and V. T. Popa, New J. Chem., 2017, 41, 15003-15015.

29. A. Shamsi, A. Ahmed, M. S. Khan, M. Al Shahwan, F. M. Husain and B. Bano, J. Mol. Liq., 2020, 311, $113348-113357$.

30. S. Zhang, R. Gan, L. Zhao, Q. Sun, H. Xiang, X. Xiang, G. Zhao and H. Li, Spectrochim. Acta A Mol. Biomol. Spectrosc., 2021, 246, 119040-119051.

31. N. A. Alsaif, T. A. Wani, A. H. Bakheit and S. Zargar, Int. J. Biol. Macromol., 2020, 165, 2451-246.

32. L. Aricov, D. G. Angelescu, A. Băran, A. R. Leontieş, V. T. Popa, A. Precupaş, R. Sandu, G. Stîngă and D.-F. Anghel, J. Biomol. Struct. Dyn., 2020, 38, 2659-2671.

33. A. Bujacz, Acta Cryst., 2012, D68, 1278-1289.

34. M. J. Frisch, G. W. Trucks, H. B. Schlegel, G. E. Scuseria, M. A. Robb, J. R. Cheeseman, G. Scalmani, V. Barone, B. Mennucci, G. A. Petersson, H. Nakatsuji, M. Caricato, X. Li, H. P. Hratchian, A. F. Izmaylov, J. Bloino, G. Zheng, J. L. Sonnenberg, M. Hada, M. Ehara, K. Toyota, R. Fukuda, J. Hasegawa, M. Ishida, T. Nakajima, Y. Honda, O. Kitao, H. Nakai, T. Vreven, J.
A. Montgomery, Jr., J. E. Peralta, F. Ogliaro, M. Bearpark, J. J. Heyd, E. Brothers, K. N. Kudin, V. N. Staroverov, R. Kobayashi, J. Normand, K. Raghavachari, A. Rendell, J. C. Burant, S. S. Iyengar, J. Tomasi, M. Cossi, N. Rega, J. M. Millam, M. Klene, J. E. Knox, J. B. Cross, V. Bakken, C. Adamo, J. Jaramillo, R. Gomperts, R. E. Stratmann, O. Yazyev, A. J. Austin, R. Cammi, C. Pomelli, J. W. Ochterski, R. L. Martin, K. Morokuma, V. G. Zakrzewski, G. A. Voth, P. Salvador, J. J. Dannenberg, S. Dapprich, A. D. Daniels, O“. Farkas, J. B. Foresman, J. V. Ortiz, J. Cioslowski and D. J. Fox, Gaussian 09, Revision C.01, Gaussian, Inc., Wallingford, CT, 2009.

35. O. Trott and A. J. Olson. J Comput Chem., 2010, 31, 455-461.

36. G. M. Morris, R. Huey, W. Lindstrom, M. F. Sanner, R. K. Belew, D. S. Goodsell and A. J. Olson, J. Comput. Chem., 2009, 30, 2785-2791.

37. Dassault Systèmes BIOVIA, Discovery Studio, San Diego: Dassault Systèmes, 2019.

38. M. J. Abraham, T. Murtola, R. Schulz, S. Páll, J. C. Smith, B. Hess and E. Lindah, SoftwareX, 2015, 1-2, 1925.

39. S. Pronk, S. Páll, R. Schulz, P. Larsson, P. Bjelkmar, R. Apostolov, M. R. Shirts, J. C. Smith, P. M. Kasson, D. van der Spoel, B. Hess and E. Lindahl, Bioinformatics, 2013, 29, 845-854.

40. N. Schmid, A. P. Eichenberger, A. Choutko, S. Riniker, M. Winger, A. E. Mark and W. F. van Gunsteren, Eur Biophys J, 2011, 40, 843-856.

41. A. K. Malde, L. Zuo, M. Breeze, M. Stroet, D. Poger, P. C. Nair, C. Oostenbrink and A. E. Mark, J. Chem. Theory Comput., 2011, 7, 4026-4037.

42. H. J. C. Berendsen, J. P. M. Postma, W. F. van Gunsteren and J. Hermans, "Intermolecular Forces", B. Pullman, Reidel, Dordrecht, 1981, p. 331-342.

43. R. Martonak, A. Laio and M. Parrinello, Phys. Rev. Lett., 2003, 90, 075503-075507.

44. M. Kawata and U. Nagashima, Chem. Phys. Lett., 2001, 340, 165-172.

45. The PyMOL Molecular Graphics System, Version 2.0 Schrödinger, LLC.

46. E. F. Pettersen, T. D. Goddard, C. C. Huang, G. S. Couch, D. M. Greenblatt, E. C. Meng and T. E. Ferrin, J. Comput. Chem. 2004, 25, 1605-1612. 